\title{
Failure Monitoring of E-Glass/Vinylester Composites Using Fiber Grating Acoustic Sensor
}

\author{
A. I. AZMI ${ }^{1,3 *}$, RAJU ${ }^{2}$, and G. D. $\mathrm{PENG}^{1}$ \\ ${ }^{1}$ School of Electrical Engineering and Telecommunications, The University of New South Wales, NSW, 2052, Australia \\ ${ }^{2}$ School of Mechanical Engineering, The University of New South Wales, NSW, 2052, Australia \\ ${ }^{3}$ Faculty of Electrical Engineering, Universiti Teknologi Malaysia, Skudai, Johor, 81310, Malaysia \\ *Corresponding author: A. I. AZMI E-mail: asrul@fke.utm.my
}

\begin{abstract}
This paper reports an application of an optical fiber sensor in a continuous and in situ failure testing of an E-glass/vinylester top hat stiffener (THS). The sensor head was constructed from a compact phase-shifted fiber Bragg grating (PS-FBG). The narrow transmission channel of the PS-FBG is highly sensitive to small perturbation, hence suitable to be used in acoustic emission (AE) assessment technique. The progressive failure of THS was tested under transverse loading to experimentally simulate the actual loading in practice. Our experimental tests have demonstrated, in good agreement with the commercial piezoelectric sensors, that the important failures information of the THS was successfully recorded by the simple intensity-type PS-FBG sensor.
\end{abstract}

Keywords: Fiber acoustic sensors, intensity-type, phase-shifted, acoustic emission

\section{Introduction}

Acoustic emission (AE) is a versatile and established noninvasive evaluation technique, which has been used for many purposes including structural failure detection and process control. This technique can be used to gather internal structural information without requiring any invasive procedure. Particular interest of AE application has been shown in failure assessment of composite structures, as the $\mathrm{AE}$ technique is capable of distinguishing the complex failure modes from certain waveform characteristics. Hence, all the industries that substantially use the composites such as the aerospace and marine industries, have applied this technique to studying the structural failures [1-3].

Top hat stiffener (THS) is a marine structure that is used in high performance yachts to sustain the tensile and bending load of the keel [3]. Modern yacht design requires the lighter hull, higher speed, and lower center of gravity. These demands have encouraged the use of the small keel-hull mating area, hence, generation of enormous stress concentration on the mating area. A number of keel related accidents have been witnessed recently suggesting that inferior design and flaw manufacturing process have been the culprits. Due to the extreme working condition, design enhancement at the keel-hull joint is necessary. Improvement in the THS design seems more convenient and feasible to be achieved compared to other structures such as the hull. Continuous design refinement and test cycles would lead to improved designs, subsequently, which may prevent in-service catastrophic failure that could be very costly in terms of economy and lives.

Received: 23 June 2012 / Revised version: 8 July 2012

(C) The Author(s) 2012. This article is published with open access at Springerlink.com 
This paper reports the implementation of the acoustic emission technique in failure assessment of novel E-glass/vinylester THS composites. To implement the AE method in the structural test, an optical fiber acoustic sensor system has been developed and further deployed in situ. The system is based on intensity-modulation [4-7] and consists of a phase-shifted (PS) fiber Bragg grating (FBG) as the sensing head. The narrow transmission channel (or resonance) feature of the PS-FBG is highly sensitive to small perturbations, hence suitable for measuring the acoustic wave. The sensitivity of a simple intensity-type PS-FBG acoustic sensor was manifested in a continuous structural failure monitoring based on the $\mathrm{AE}$ technique. The progressive failure was monitored from a THS that underwent transverse loading, which resembled to the actual load seen in practice. The released acoustic waves from the progressive failure were continually recorded using a surface attached PS-FBG, placed close to the strategic point that was likely to fail. Throughout the course of the test, the PS-FBG sensor successfully detected the important failures information of the THS. The result was in close agreement with the commercial piezoelectric sensors.

\section{Failure assessment of composites based on the acoustic emission technique}

\subsection{Acoustic emission method and failure signatures in composites}

$\mathrm{AE}$ from the non-destructive evaluation (NDE) sense can be defined as the phenomena where transient elastic waves are generated by rapid release of energy from localized sources within a material. $\mathrm{AE}$ techniques are capable of resolving crucial information including real-time damage activity, damage position identification, damage type identification, and strength predictions to assess the residual structural strength. The AE technique is applicable to both global and local methods, whereby a single $\mathrm{AE}$ sensor can be used to monitor the large structure area or within the concentrated area, respectively. Each of the failure mode produces certain acoustic signatures distinguished from one to another. Among the typical AE parameters used to assess the failure modes in composites are the amplitude, risetime, counts, and threshold. Other variables such as sensors, couplant, material and dimension, also give rise to the differences of the failure signatures.

\subsection{THS dimension and compositions}

The dimension of the top hat stiffener used in this study is shown in Fig. 1. Each specimen was $100 \mathrm{~mm}$ wide and $200 \mathrm{~mm}$ high. The crown was $200 \mathrm{~mm}$ long with radii of $19 \mathrm{~mm}$ and $23 \mathrm{~mm}$ at top and bottom bends, respectively. Table 1 shows fiber types used in manufacturing the THS. The layer number indicates the stacked sequence, with the lowest denoting the bottom layer. The final sample thickness may vary depending on laminating procedure used. Basic material properties of the E-glass fiber used can be found in [3].

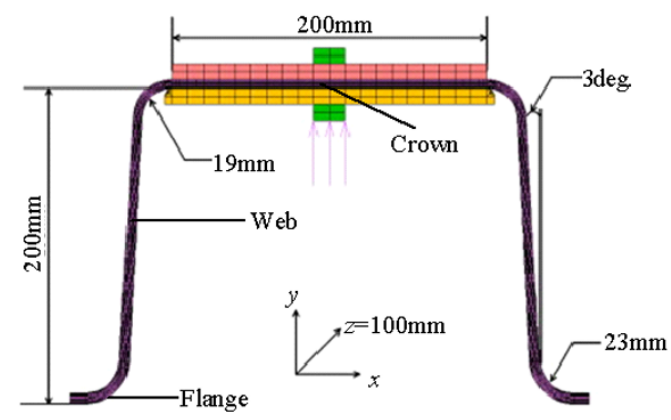

Fig. 1 Dimension of the top hat stiffener.

Table 1 Top hat stiffener composition.

\begin{tabular}{ccc}
\hline Layer & Fiber type & Thickness $(\mathrm{mm})$ \\
\hline 8 & DB450 & 0.26 \\
7 & DB450 & 0.26 \\
6 & CSM450 & 0.6 \\
5 & DB450 & 0.26 \\
4 & CSM450 & 0.6 \\
3 & DB450 & 0.26 \\
2 & CSM450 & 0.6 \\
1 & DB450 & 0.26 \\
\hline
\end{tabular}

DB450: E-glass double bias $451 \mathrm{~g}$.

CSM450: E-glass chopped strand mat $451 \mathrm{~g}$. 


\section{Experiment}

\subsection{Experiment setup}

An in-house optical fiber acoustic measurement system has been constructed as shown in Fig. 2. It is a typical intensity-type acoustic measurement system consisting of a tunable laser system as the interrogation source and a fiber grating-based sensor. In this work, a PS-FBG was used as the sensing head distinguished from the previous reported system that employed the normal FBG as the sensing head [5-7]. Four piezoelectric sensors were also employed to provide the reference measurement.
A THS specimen was tested under configuration shown in Fig. 3. Both flanges were clamped onto the stage, while the crown was centrally bolted to the loading device, with two steel plates sandwiching the crown to reproduce the typical loading condition. The load was applied upward, in the transverse direction of the THS surface. The pull-up deflection rate was set at $2 \mathrm{~mm} / \mathrm{min}$. The deflection and load were measured by the extensometer and load cells, respectively, which were equipped with the Instron test machine. The load-deflection plots were monitored using Bluehill software.

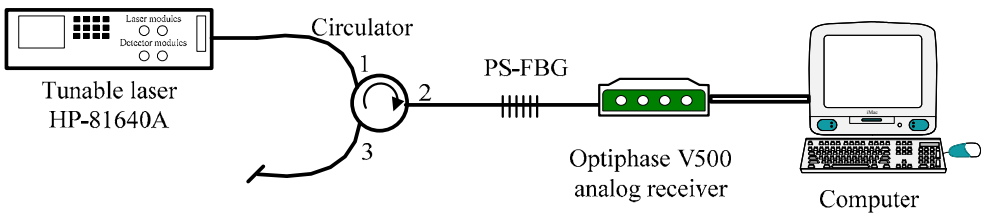

Fig. 2 FBG acoustic measurement system.

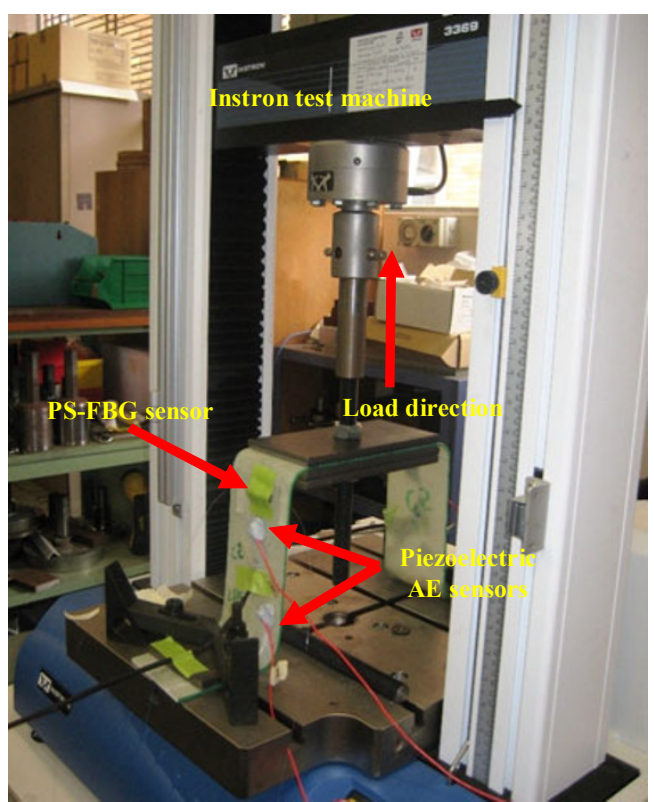

Fig. 3 Experimental configuration.

A PS-FBG is known to have the narrow transmission channel or resonance at the central wavelength. With the high slope of the reflectivity and narrow bandwidth of the transmission spectral, it is expected that this sensing head possesses higher sensitivity compared to the normal FBG. With high sensitivity, the sensor can be deployed on the structure surface using soft contact of ultrasonic coupling gel. Under soft contact of low viscosity gel, the FBG is insensitive to quasi-static strain effect. Room temperature was maintained throughout the experiment. Constant reading of the DC voltage implied that the preset operating point was constant throughout the measurement. With such temperature stability, measurement could be taken over the extended period of time without adjustment of the laser's wavelength, thus, simplifying the overall system. An air gap between the sensor and specimen was eliminated to enhance acoustic coupling efficiency [8]. A polyolefin tube was longitudinally cut into the half-cylinder shape to affix the gel and sensor together on the vertical surface of the specimen.

Due to the narrow bandwidth and high optical power, the power spectrum of the laser diode is very focused at a particular wavelength. Hence, the relative sensitivity can be calculated directly from the reflectivity steepness of the sensing FBG [4]. The transmission spectrum of the PS-FBG is shown in Fig. 4(a). The PS-FBG was fabricated with 8-mm 
length, and no apodization profile was applied. A centrally located $\pi$-phase shift created a narrow transmission band $(\sim 10 \mathrm{pm}$ at $-3 \mathrm{~dB}$ point and centered at $1556.13 \mathrm{~nm})$. For acoustic measurement, the laser wavelength was adjusted to be slightly offset from the resonance. The best offset point that compromised both the sensitivity and dynamic range was $26 \mathrm{pm}$, and the maximum offset was $60 \mathrm{pm}$. The pressure range with a 26-pm offset also signified a pressure range of $7.5 \mathrm{MPa}$, which could be attained from the uncoated FBG response. The peak power incident on the sensor was $2 \mathrm{~mW}$. Assume the pressure-wavelength sensitivity of bare fiber is $3.47 \times 10^{-6} \mathrm{pm} \cdot \mathrm{Pa}^{-1}$. From the power spectrum, the average rate of power change per wavelength was about $0.035 \mathrm{~mW} \cdot \mathrm{pm}^{-1}$. As the resonance of the PS-FBG was shifted due to perturbation, the transmitted power was given by a rate of about $0.12 \mathrm{pW} \cdot \mathrm{Pa}^{-1}$. The receiver gain used was $2 \mathrm{mV} \cdot \mathrm{W}^{-1}$, hence the rate of voltage change per pressure was about $0.24 \mu \mathrm{V} \cdot \mathrm{Pa}^{-1}$. A national instrument PCI-9250 data acquisition (DAQ) card was used to interface the computer to the photodetector. Given that the DAQ card input sensitivity was $6 \mu \mathrm{V}$, the minimum detectable pressure was about $148 \mathrm{~dB} \cdot \mathrm{re} \cdot 1 \mu \mathrm{Pa}$. A circulator was used as the interface between the light source and sensor, rather than direct transmission to prevent the unused reflected light from destabilizing the laser diode. An example of a transmission spectrum of a fabricated normal FBG is illustrated in Fig. 4(b). The FBG was written with 20-mm length, cosine apodized and had 0.1-nm FWHM bandwidth. The linear transition region occurred from $-3 \mathrm{~dB}$ point to $-20 \mathrm{~dB}$ point. Although with the length of more than twice of the PS-FBG, the slope of the transmission spectrum of the FBG is about 8 times lower than that of the PS-FBG. This explains the advantages of the PS-FBG compared to the normal FBG. The sensitivity and frequency response of PS-FBG sensor were further put into tests before being utilized in the practical application.

The computer used for acoustic monitoring was equipped with a Pentium 4 processor, $256 \mathrm{MB}$ of RAM, and 7200 RPM IDE hard drive. The main challenges in developing the data acquisition system is to continuously capture high frequency signals, therefore, focus of data acquisition is more towards

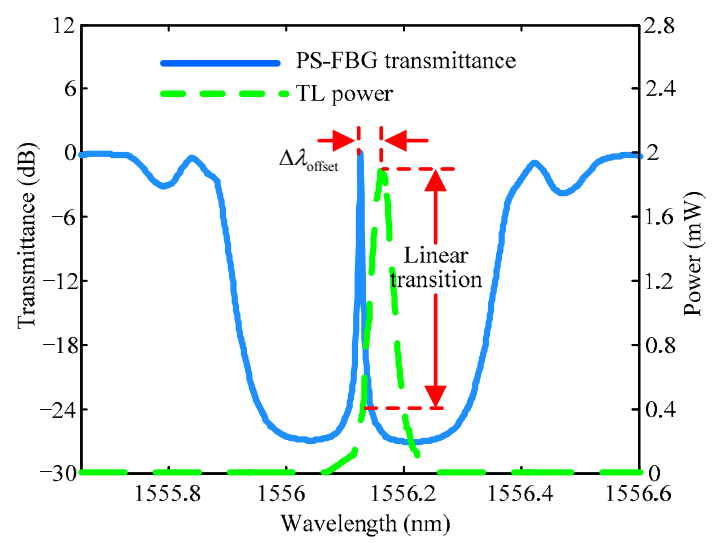

(a)

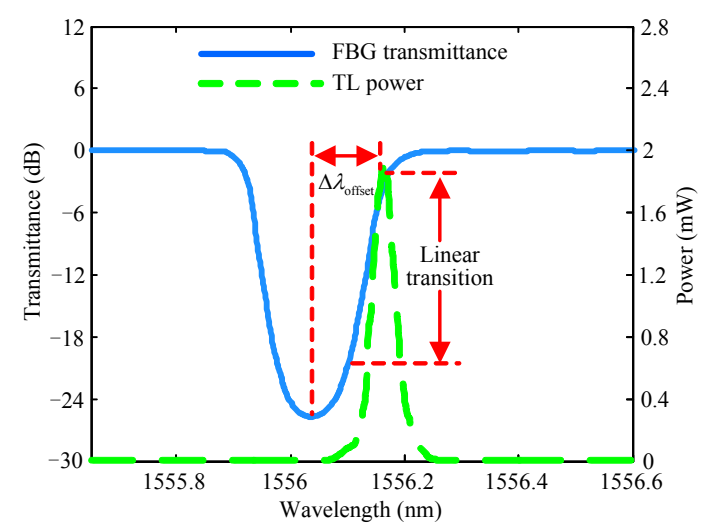

(b)

Fig. 4 Biasing condition of a TLS lasing spectrum with respect to (a) transmission spectrum of the PS-FBG (used in the experiment) and (b) transmission spectrum of the normal FBG. optimization of software flow. For acoustic sensing monitoring, the software needs to record the waveform continuously, with time resolution ranging from microsecond unit to nanosecond unit (to resolve the high frequency signal). This requirement reflects that the huge amount of data will be processed. While the software flow needs to be efficient, the complex task such as the real-time digital signal processing (DSP) and data recording can be implemented at the optimal capability of the computer and DAQ card. Offline data processing will be much easier using the automated software. 
The software must be able to perform correctly the parameters extraction from the recorded waveform. The voltage range of DAQ card input set was from $-5 \mathrm{~V}$ to $5 \mathrm{~V}$ which was similar to the voltage range of the Optiphase V-500 receiver. The analog data was sampled at the highest sampling frequency, $f_{s}$ of DAQ at $1 \mathrm{MS} / \mathrm{s}$ correspondingly to the highest detected frequency, $f_{m}$ of $500 \mathrm{kHz}$. The buffer size of the card was set at 15 bits, resulting in $2^{15}=$ 32768 number of points for the recorded waveform. With respective settings, the achieved time and frequency resolution in measurement were $\Delta t=1 / f_{s}=$ $1 \mu \mathrm{s}$ and $30.5 \mathrm{~Hz}$, respectively. Each duration of each recorded waveform had duration of $\Delta t \times 32768 \approx$ $32.8 \mathrm{~ms}$. The implemented data acquisition process utilizing the Labview software is shown in Fig. 5. The software coordinated the execution of the loop 4 times in a second to allow digital signal processing and parameters recording could be completed in one cycle, hence the time spacing between data sets was $0.25 \mathrm{~s}$ apart. The time gap was essential to ensure the stability of the real-time acquisition although it was comparatively much larger than the duration of a waveform. In a normal loop, each digital waveform was filtered using the finite impulse response (FIR) high pass filter (HPF) with a cutoff frequency $f_{\text {co }}$ of $2 \mathrm{kHz}$. This would remove unwanted DC and low frequency components. The essential parameters such as amplitude, rms (or effective value) and time were then extracted and recorded in real time and in situ. The waveform was recorded only if the amplitude exceeded the threshold voltage to minimize real-time processing load and also reduce the waveform file size that later could be much easier in offline parameters extraction. The waveform can be also saved if the user manually invokes the save function.

The reference measurement used in this study was the $\mathrm{AE}$ equipment from Physical Acoustic Corp. (PAC). The piezoelectric sensors came with a complete set of $\mathrm{AE}$ equipment for real-time monitoring and post-processing. This commercial
$\mathrm{AE}$ equipment effectively recorded the stress waves and then automatically analyzed AE parameters. It had four channels with the 16-bit high-speed analog to digital (A/D) converters and high-end multiple digital signal processor technology on a single PCI card. The high processing capability allowed the system to assess progressive real-time $\mathrm{AE}$ features in both the time and frequency domains. The "Nano 30 " piezoelectric sensors from PAC, with a resonant frequency of $140 \mathrm{kHz}$, were used as the sensor head. During experiment, four piezoelectric sensors were affixed on the surface of both vertical sides of the specimen that was close to the top and the bottom corners. A blu-tack adhesive in conjunction of a "2211-silicone compound" (geophysical grade/high vacuum grease) were used as a constant holder and acoustic couplant, respectively. AE was recorded on a personal computer laptop using "AEwin" software for DiSP system, which was commercially available software from PAC. Data from piezoelectric sensors was extracted using the commercial software WinPost from the PAC. All the sensors were tested using the available automatic sensor test (AST) function to ensure comparable data recorded by sensors of the same type. The standard channel setup and the signal-processing filter for the DiSP equipment were set as recommended by the manufacturer.

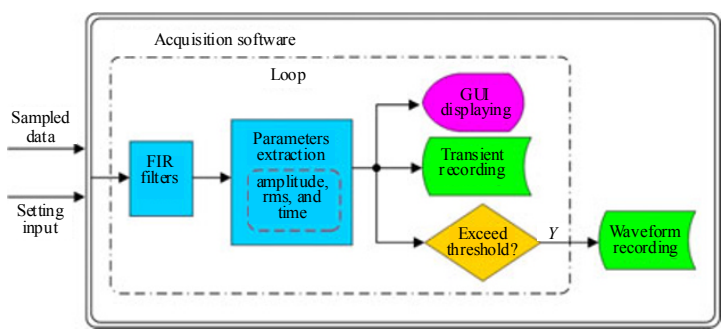

Fig. 5 Real-time digital signal processing flow.

\subsection{Result and discussion}

Failure modes were assessed from several parameters extracted from the recorded waveform of the released stress waves, including the amplitude, duration, risetime, energy, counts and cumulative counts. Shown in Table 2 are the parametric values 
and frequency range of the different failures modes that have been established based on our extensive literature survey [9].

Table 2 THS damage signatures [9].

\begin{tabular}{cccccc}
\hline \multirow{2}{*}{ Failure Mode } & Amplitude & Energy & \multicolumn{3}{c}{ Duration Risetime Frequency } \\
& $(\mathrm{dB})$ & $(\mathrm{e} \mu \mathrm{J})$ & $(\mathrm{ms})$ & $(\mu \mathrm{s})$ & $(\mathrm{kHz})$ \\
\hline Matrix cracking & $40-55$ & $<5000$ & $8-10$ & N/A & $50-150$ \\
\hline Interface debonding & $55-65$ & $5000-8000$ & $10-15$ & $1-5$ & $150-350$ \\
\hline Fiber breakage & $65-85$ & $9000-12000$ & $>15$ & $5-20$ & $350-400$ \\
\hline Delamination & $90-100$ & $>12000$ & $>25$ & $>20$ & $>450$ \\
\hline Fiber pull-out & $65-85$ & N/A & N/A & N/A & $0-250$ \\
\hline
\end{tabular}

Figures 6 and 7 show the plots of the amplitude and load versus loading time for piezoelectric and PS-FBG sensors, respectively. The amplitude plot for the piezoelectric was narrowed in the range between $60 \mathrm{~dB}$ and $100 \mathrm{~dB}$, as the amplitude level below $60 \mathrm{~dB}$ was dominated by noise. On the other hand, for the PS-FBG sensor, the amplitude range was set between $0 \mathrm{~dB}$ and $40 \mathrm{~dB}$ which was comparable to the range of the piezoelectric sensor, which was $40 \mathrm{~dB}$. It could be seen that the amplitude measurement of the PS-FBG was highly correlated to the piezoelectric sensors, whereby both sensors exhibited high amplitude excursions at the time when the main failures occurred. From observation of the amplitude excursion, there were 11 main failures occurring throughout the loading procedure at $214 \mathrm{~s}, 294 \mathrm{~s}, 314 \mathrm{~s}, 339 \mathrm{~s}, 377 \mathrm{~s}, 416 \mathrm{~s}, 486 \mathrm{~s}, 524 \mathrm{~s}$, $590 \mathrm{~s}, 649 \mathrm{~s}$, and $737 \mathrm{~s}$. The amplitude excursions

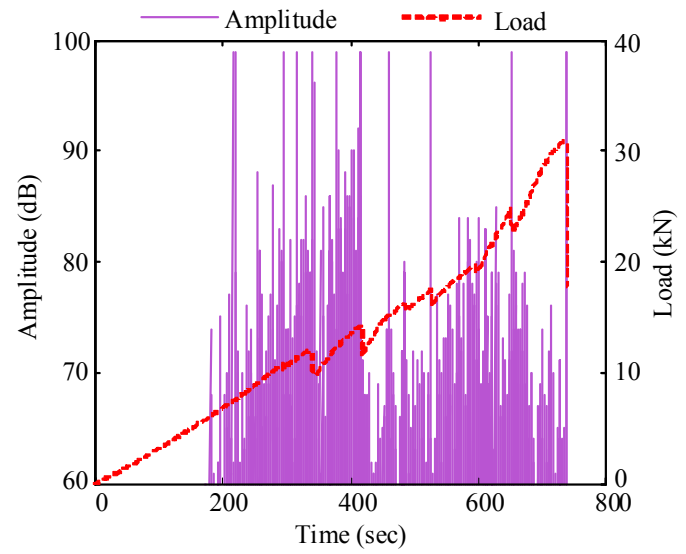

Fig. 6 Amplitude vs. time, measured by the piezoelectric sensor. were also coincident with the load changes, which are shown on the right hand side of the $y$-axis. The load drops implied the structure temporarily lost the resistance/ strength to the applied force.

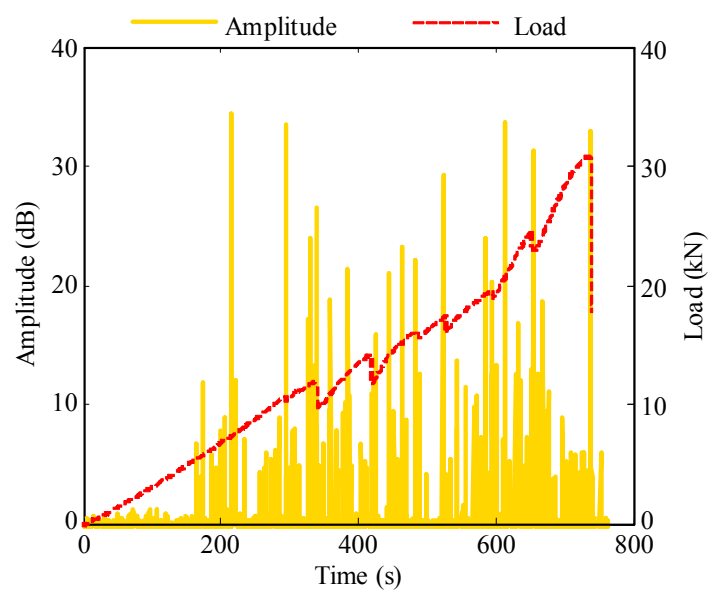

Fig. 7 Amplitude vs. time, measured by the PS-FBG sensor.

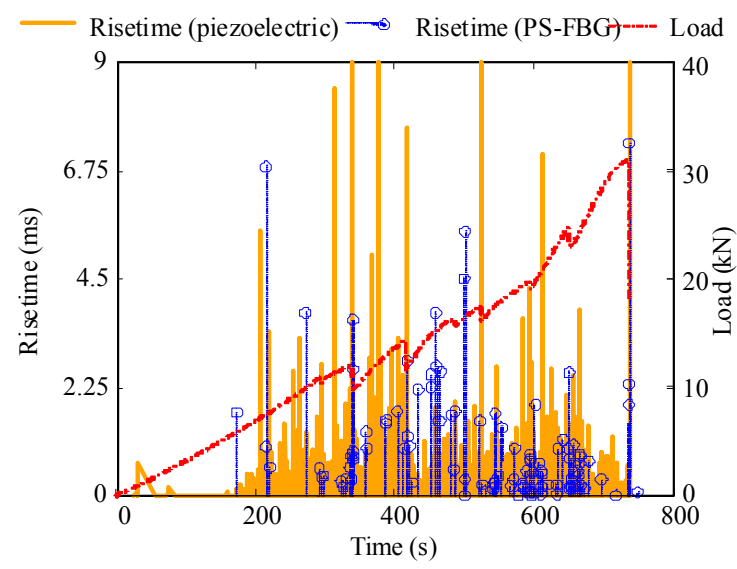

Fig. 8 Comparison of the risetime parameter extracted from the PS-FBG sensor and piezoelectric sensor data.

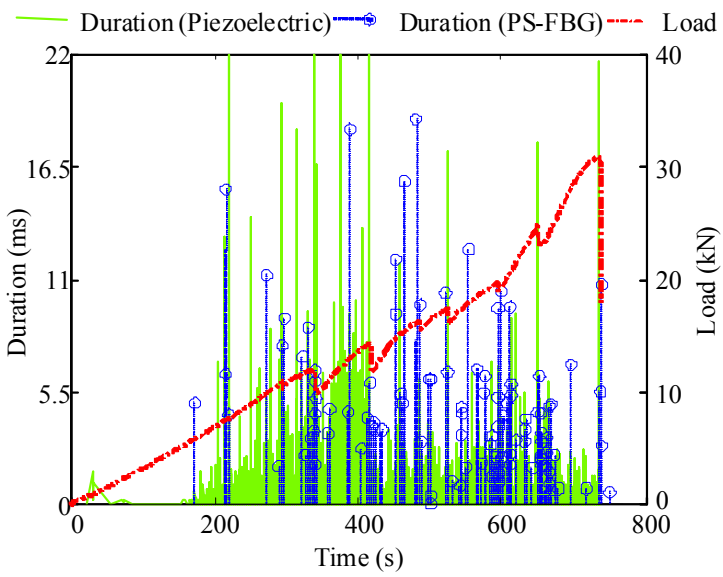

Fig. 9 Comparison of the duration parameter extracted from the PS-FBG sensor and piezoelectric sensor data. 
Figures 8 and 9 show the plots of the risetime and duration of the PS-FBG compared to those of the piezoelectric sensor. Results for the duration and risetime parameters showed noticeable discrepancies compared to the piezoelectric sensor. These discrepancies were attributed to the different levels of the technical ability between the employed sensor systems. The acoustic measurement from the piezoelectric sensors was used to establish the failure modes due to its reliability. The first failure at $214 \mathrm{~s}$ was expected to be matrix cracking, with a small drop in the load of $7 \mathrm{~N}$, but with a high energy release and duration of $5914 \mathrm{eu}\left(1 \mathrm{eu}=10^{-18} \mathrm{~J}\right)$ and $25.80 \mathrm{~ms}$. The major crack that occurred at $294 \mathrm{~s}$ was a delamination failure with a significant drop in the load of $484 \mathrm{~N}$. The signal lasted $18.88 \mathrm{~ms}$, and the energy released was $6549 \mathrm{eu}$. The signal at $320.33 \mathrm{~s}$ corresponded to crack propagation with a $437-\mathrm{N}$ drop in the load, but the risetime and counts were just $0.56 \mathrm{~ms}$ and 467 . A large energy release of 10,358 eu was observed at $422.50 \mathrm{~s}$ with a load drop of $1,575 \mathrm{~N}$, corresponding to a delamination failure. The duration and counts were $68.51 \mathrm{~ms}$ and 3039 , respectively. Further loading caused the cracks to propagate in the laminate; three peak values were observed at $486.50 \mathrm{~s}, 524 \mathrm{~s}$, and $590 \mathrm{~s}$. The signal duration was smaller compared to the delamination signal. Another major crack occurred at $649 \mathrm{~s}$ with a $1122-\mathrm{N}$ drop in the load. The parametric values of the energy, duration, risetime, and counts were $5804 \mathrm{eu}, 17.70 \mathrm{~ms}, 0.86 \mathrm{~ms}$, and 347, respectively. Final collapse was assumed to be fiber failure where a huge drop in the load of $12,457 \mathrm{~N}$ was observed.

Detail inspection was performed on the recorded waveforms originated from the major failures to validate the result obtained by the PS-FBG. The recorded waveforms from the PS-FBG were analyzed for their AE parameters using in-house software analysis tools. Tables 3 and 4 outline the main parameters of the waveforms recorded by piezoelectric and PS-FBG sensors, respectively. Since the sensor unexpectedly exhibited lower sensitivity at the higher frequency (due to the couplant), the fast Fourier transform (FFT) of the PS-FBG was divided into two regions: below $20 \mathrm{kHz}$ and above $20 \mathrm{kHz}$, as the PS-FBG. By separating the FFT into two regions, the peak frequency of the acoustic signal above $20 \mathrm{kHz}$ was more recognizable. The PS-FBG detected exactly the same peak frequency of the piezoelectric sensor for failures of no. 3 and 5. The PS-FBG also detected the almost similar frequency of the piezoelectric sensor for failures of no. 1, 8, 9, and 11. Failures of no. 2, 4, and 10 were completely undetected since the signal frequencies were beyond the detectable range of the PS-FBG sensor. While for failure of no. 7, there was no data available from the piezoelectric sensor. The recorded waveforms for failures of no. 1 and 3 are shown in Figs. 10 and 11, respectively.

Table 3 Amplitude and peak frequency of FFT obtained from the piezoelectric sensors.

\begin{tabular}{cccc}
\hline No. & Amplitude $(\mathrm{mV})$ & Peak FFT $(\mathrm{kHz})$ & Failure mode \\
\hline 1 & 0.49 & 55.90 & Matrix cracking \\
\hline 2 & 9.61 & 647.61 & Delamination \\
\hline 3 & 0.24 & 4.61 & Crack progression \\
\hline 4 & 9.91 & 874.37 & Delamination \\
\hline 5 & 0.25 & 8.79 & Crack progression \\
\hline 6 & 0.20 & 4.36 & Delamination \\
\hline 7 & N/A & N/A & Crack progression \\
\hline 8 & 1.23 & 87.69 & Crack progression \\
\hline 9 & 0.76 & 26.50 & Crack progression \\
\hline 10 & 9.90 & 617.55 & Delamination \\
\hline 11 & 1.21 & 61.48 & Fiber break \\
\hline
\end{tabular}

Table 4 Amplitude and peak frequency of FFT obtained from PS-FBG acoustic sensor.

\begin{tabular}{cccc}
\hline No. & Amplitude (mV) & $\begin{array}{c}\text { Peak FFT } \\
\text { (below 20 kHz) }\end{array}$ & $\begin{array}{c}\text { Peak FFT } \\
\text { (above 20 kHz) }\end{array}$ \\
\hline 1 & 150.7 & 5.951 & 55.05 \\
\hline 2 & 33.11 & 5.157 & 20.81 \\
\hline 3 & 12.34 & 4.883 & 73.67 \\
\hline 4 & 13.33 & 14.95 & 137.70 \\
\hline 5 & 9.247 & 7.446 & NA \\
\hline 6 & 40.98 & 2.136 & 73.67 \\
\hline 7 & 35.16 & 7.507 & 24.6 \\
\hline 8 & 57.42 & 3.265 & 77.4 \\
\hline 9 & 54.27 & 5.157 & 24.7 \\
\hline 10 & 12.45 & 7.599 & 20.9 \\
\hline 11 & 60.26 & 6.104 & 57.1 \\
\hline
\end{tabular}




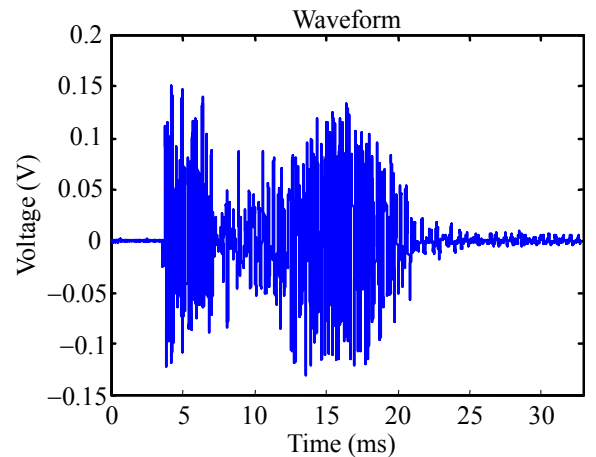

(a)

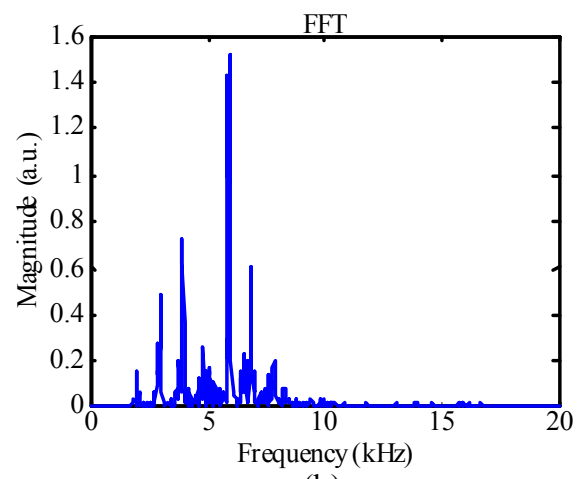

(b)

Fig. 10 Acoustic waveform of failure no. 1 recorded by the PS-FBG sensor: (a) the waveform and (b) the corresponding FFT.

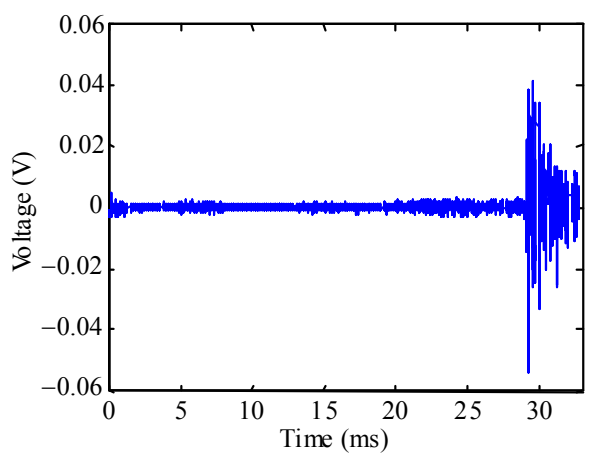

(a)

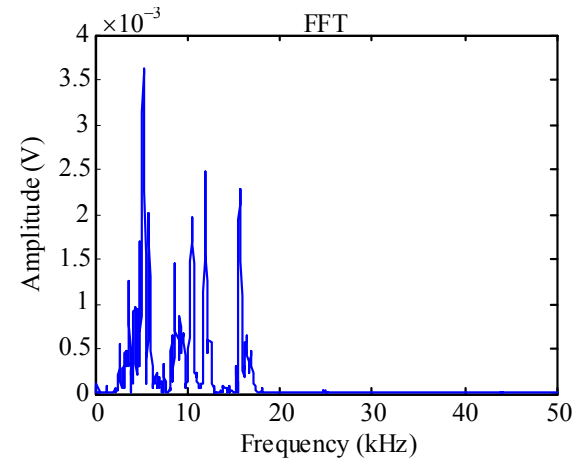

(b)

Fig. 11 Acoustic waveform of failure no. 3 recorded by the PS-FBG sensor: (a) the waveform and (b) the corresponding FFT.
In this preliminary test, it has been noted that the developed PS-FBG sensor system was excellent in term of amplitude measurement which was comparable to the amplitude obtained from the piezoelectric sensor. Hence, the PS-FBG sensor has demonstrated sufficient sensitivity acquiring the acoustic wave during the test. For future works, the focus will be given to the improvement of the data acquisition rate, couplant, sensor frequency response, and operating point stability of the system. For the current PS-FBG sensor, the recording rate is limited to 4 times in one second, while the waveform duration is $32.8 \mathrm{~ms}$ for the PS-FBG sensor. Some of acoustic waves may not be completely recorded, and hence the extracted parameters from the waveform will be dissimilar as well. The result obtained by the piezoelectric sensor was a combined result from 4-unit piezoelectric sensors deployed close to each critical point. In contrast, only 1 unit of the PS-FBG sensor was deployed on one of the critical points. This factor also affected the amplitude and other waveform characteristics, since the acoustic propagation loss was enhanced by the distance. While for a complete success in implementation, may require further improvement in the areas mentioned above. Nonetheless, this research work was an important step in understanding the development process of the optical fiber-based $\mathrm{AE}$ assessment technique.

\section{Conclusions}

This paper reports the application of PS-FBG acoustic sensors for failure test and assessment of E-glass/vinylester top hat stiffener composites based on the acoustic emission characteristics. With a narrow transmission band, the PS-FBG is highly sensitive to small perturbations from acoustic emissions associated with the failure process of the THS. Such capability has been experimentally demonstrated in continuous in situ AE testing, whereby the amplitude measurement has demonstrated a good agreement with the 
piezoelectric sensors. Detail inspection that performed on the recorded waveforms originated from the major failures has further validated the result obtained by the PS-FBG in term of the detected frequency of the waveform. As the $\mathrm{AE}$ method is very demanding in terms of the acquisition speed and signal processing, there are a few issues subjected to further investigation, and the improvement have been noted.

Open Access This article is distributed under the terms of the Creative Commons Attribution License which permits any use, distribution, and reproduction in any medium, provided the original author(s) and source are credited.

\section{References}

[1] K. M. Holford, R. Pullin, S. L. Evans, M. J. Eaton, J. Hensman, and K. Worden, "Acoustic emission for monitoring aircraft structures," Journal of Aerospace Engineers, vol. 223, no. 5, pp. 525-532, 2009.

[2] G. Wang, M. Lee, C. Serratella, S. Botten, S. Ternowchek, D. Ozevin, et al., "Testing of acoustic emission technology to detect cracks and corrosion in the marine environment," Journal of Ship Production, vol. 26, no. 2, pp. 106-110, 2010.

[3] Raju, B. G. Prusty, D. W. Kelly, D. W. Kelly, and G. D Peng, "Top hat stiffeners: a study on keel failures," Ocean Engineering, vol. 37, no. 13, pp. 1180-1192, 2010.

[4] I. Azmi, D. Sen, W. Sheng, J. Canning, and G. D. Peng, "Performance enhancement of vibration sensing employing multiple phase-shifted FBG," Journal of Lightwave Technology, vo. 29, no. 22, pp. 3453-3460, 2011.

[5] D. J. Webb, J. Surowiec, M. Sweeney, D. A. Jackson, L. Gavrilov, J. W. Hand, et al., "Miniature fiber optic ultrasonic probe," in Proc. SPIE, vol. 2839, pp. 76-80, 1996.

[6] G. Wild and S. Hinckley, "Fiber Bragg grating acoustic emissions and transmission sensor in carbon fiber composites," in Proc. SPIE, vol. 7268, pp. 726814, 2008.

[7] I. Perez, H. L. Cui, and E. Udd, “Acoustic emission detection using fiber Bragg gratings," in Proc. SPIE, vol. 4328, pp. 209-215, 2001

[8] P. Theobald, B. Zeqiri, and J. Avison, "Couplants and their influence on AE sensor sensitivity," Journal of Acoustic Emission, vol. 26, pp. 91-97, 2008.

[9] Raju, A. I. Azmi, and B. G. Prusty, "Acoustic emission techniques for failure characterisation in composite top-hat stiffeners," Journal of Reinforced Plastics and Composites, vol. 31, no. 7, pp. 495-516, 2012. 